\title{
System Identification and Model Predictive Control of the Chatter Phenomenon in Turning Process
}

\author{
Atiyeh Karimipour ${ }^{1}$, Mohsen Emami ${ }^{1}$ \\ 1 Behbahan Khatam Alanbia University of Technology, Iran \\ * Corresponding author's e-mail: karimipour@aftermail.ir
}

\begin{abstract}
Chatter is a series of unwanted and extreme vibrations which frequently happens during different machining processes and impose variety of adverse effects on the machine-tool and surface finish. Chatter has two main types namely forced-chatter and self-existed chatter. The forced-chatter has an external cause; however, self-exited chatter has no external stimuli, rather it is created due to the phase difference between the previous and current waves on the surface of the workpiece. Due to the self-generative nature of this type of chatter, its recognition and prevention is much more difficult. For preventing self-exited chatter its model should be available first. The chatter is usually simulated as a one degree of freedom mass-spring-damper model with unknown parameters that they should be determined somehow. In this paper, the parameters of the tool equation of motion i.e. mass, damping, and stiffness coefficients of the system are predicted through a wavelet-based method online, and then based on the achieved parameters, the system is controlled via Model Predictive Control (MPC) approach. For the validation, the algorithm is applied to 25 different experimental tests in which the acceleration of the tool and cutting force are measured via an accelerometer and a dynamometer. By investigation of the SLDs generated by the predicted parameters, the presented system identification method is validated. Also, it is shown that the chatter vibration is completely restrained by means of MPC. For investigation of the MPC performance, MPC algorithm is compared with PID controller and simulations has indicated a much stronger performance of MPC rather than PID controller in terms of vibration attenuation and control effort.
\end{abstract}

Keywords: turning process, chatter phenomenon, system identification, discrete wavelet transform, and model predictive control

\section{INTRODUCTION}

Turning is one of the oldest and most adaptive procedures for production of circular components with just one cutting tool. The products which are usually created by turning process has a wide range from tiny screws for glasses to huge shafts in hydroelectric power plants. The turning is frequently conducted by a lathe in which the work piece is attached to the spindle from one side and the tailstock from the other side. The cutting tool is moved through a line, parallel or vertical to the workpiece and is subjected to a linear displacement while the workpiece is rotating.

As mentioned, during turning process, the tool and workpiece contacts with each other and a mutual force is created in the contact area. Some- times, this dynamical force leads to unwanted vibrations called self-excited chatter. The self-excited or regenerative chatter is produced because the machined surface is wavy which generates a vibrating cutting force in the next rotation, in addition this vibrating force itself creates a wavy surface too. This process regenerates itself because the cutting force always vibrates due to the previous wavy surface. The relative movement between the tool and workpiece will continue until the chatter is fully developed and the final machined surface will be completely wavy [1].

On the other hand, there is another type of chatter called forced-chatter. It occurs when an external force motivates the structure. The self-generative chatter can usually be prevented through changing the cutting parameters in- 
cluding the spindle speed, depth of cut and feed rate. Whereas, the forced-chatter usually can be vanished by removing or adjusting the external force. All types of chatter have many destructive effects such as increasing cutting forces, poor surface finish, decreasing of the tool and lathe lifetime, imprecise dimensions of the product, and load noises [2].

Until now, many researchers have proposed different methods for coping with chatter. The first step for dealing with chatter is to extract a model which can appropriately describe its behavior. But, since it is not possible to predict the exact mechanics and dynamics of cutting, a complex model which can define the complete metal cutting dynamics has not been suggested yet [3]. The analysis of chatter includes modeling of the tool and workpiece interaction and modelling of the cutting force which together make a closed-loop dynamical model for turning. After derivation of a model, analytical methods are used to determine the stability status of the process. The curve which determines the stability status of the operation is called the Lobe Diagram. This diagram shows stable and unstable zones in the cutting parameters space including spindle speed and depth of cut [4]. For recognition of the stability status of a process by this method, the exact structural parameters of the system have to be specified. One of the most common methods for obtaining the structural parameters is the impulse hammer test. However, according to [5], the structural parameters attained by this method are just an estimation of the real coefficients and therefore, always an uncertainty should be considered in the derived model. Otherwise, it is possible that a set of depth of cut and spindle speed located in the stale zone leads to an unusable process because of not considering the uncertainty [6]. Therefore, for a certain turning operation especially when the surface finish has a great importance, an online system identification model along with a reliable control approach is vital for coping with system nonlinearities and other uncertainties.

There are numerous control approaches for stabilizing chatter, but in these days, the need for better controlling chatter is increasing because there is a pressure on industries for increasing of the productivity, and accuracy, and decreasing of the production costs. There are two main ways of controlling of chatter which are passive and active control approaches. In passive methods, some devices are used to absorb the surplus of the energy including vibration absorbers, friction dampers, mass-dampers or tuned dampers. These devices have frequently lower rigidity and can damp the chatter vibrations. In passive methods, dynamical vibration absorbers or tuned mass dampers are wildly utilized in practical applications in comparison with other methods. Tobias in [7], has presented several practical methods and their effect on the stability of the process in which the vibration absorber is attached to the different parts of the machine-tool. Passive control methods of chatter vibration have many advantages such as low cost, not needing any external energy and easy implementing. But, for more accurate performance the dampers in TMDs need to be tuned online like in active control approaches.

In active control methods, chatter vibrations are actively identified by continuous monitoring of the process, and when it is necessary, the required changes are applied to the system. Some researchers have proposed some techniques that the system parameters such as depth of cut and spindle speed are changed during the process in accordance with the control algorithm needs. For instance, Lin and $\mathrm{Hu}[8]$ has presented a method in which the feed rate and spindle speed are altered for vanishing chatter. Frumusanu and et al. [9], have offered a control method based on the real-time monitoring of the cutting force.

The main objectives of the present work are the parameter estimation of the system model and chatter vibrations control. For the parameter estimation, first the discrete wavelet transform of the displacement, velocity, acceleration, and force signals are calculated. Then, by putting the achieved coefficients in the system equation of motion and solving it, the mass, damping, and stiffness coefficients of the system are extracted. For controlling of the chatter phenomenon, based on the attained structural parameters, the Model Predictive Control (MPC) algorithm is applied. Finally, the performance of the MPC and PIC controllers are compared.

The main features of the proposed method are:

- For the system identification there is no need for any impulse hammer test or other previous information and the parameters are extracted online during the process based on the cutting force and acceleration signals. So, they can adapt with any new cutting conditions.

- Using Model Predictive Control algorithm, the system is controlled very accurately and with low control effort. 
In section 2, the dynamics governing the chatter phenomenon and the stability lobe diagram (SLD) are presented. In sections 3 and 4, the system identification approach and the control procedure are described. Next, in section 5, the results of applying proposed algorithm on real signals are described, and finally in section 6 , the conclusion of the present work is expressed.

\section{THE CHATTER THEORY}

The regenerative chatter forms due to the interaction between the metal cutting process and the machine-tool. This type of chatter occurs near the dominant natural frequency of the machine-tool. The excitation of this mode leads to the relative movement between the tool and the workpiece. This relative movement can be modeled as a linear one-DoF system as can be seen in Fig. 1. In this figure, $m, c$, and $k$ are the mass, damping, and stiffness coefficients of the system. Also, $y(t)$ is the wave formed during the current revolution and $y(t-\tau)$ is the wave formed during the previous workpiece revolution. The phase difference between $y(t)$ and $y(t-\tau)$ is the main cause of chatter in the turning. If these two waves have the same phase, no vibration will grow in the system because, the variations in the chip thickness will be very small and negligible. From the energy point of view, when the system is marginally stable, the produced and the dissipated energy becomes equal. Therefore, when no phase difference exists, there is no surplus of energy and so the process will be stable. On the other hand, when the waves do not have the same phase, because the entered energy to the system is more than the dissipated energy, the vibration grows on the workpiece and the system will be unstable.

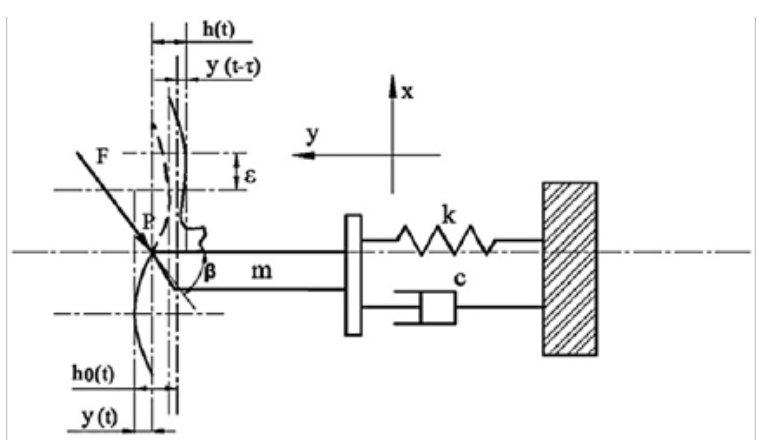

Fig. 1. One DoF mass-spring-damper model of the turning process [4]
If it is considered that the tool is flexible and the workpiece is rigid the equation of motion becomes (Fig. 1):

$$
\begin{aligned}
& m \ddot{y}(t)+c \dot{y}(t)+k y(t)=F_{f} \\
& F_{f}=K_{f} b\left[y_{0}-y(t)+y(t-\tau)\right]
\end{aligned}
$$

in which $y(t)(m)$ is the displacement of the tool along y direction. $b(\mathrm{~m})$ is the depth of cut, $\tau(s)$ is the period between two consecutive revolution, and $y(t)-y(t-\tau)(m)$ is the chip thickness which is not constant and is a variable of time. Also, $F_{f}$ is the cutting force and $K_{f}$ is the cutting coefficient along the $y$ direction [4].

By applying Laplace Transform on the left side of equation (1):

$$
G(s)=\frac{1}{m s^{2}+c s+k}
$$

$G(s)$ is achieved which is the open loop transfer function of the system. Therefore, The Laplace Transform of the closed-loop system becomes:

$$
\frac{X(s)}{X_{0}(s)}=\frac{b K_{f} G(s)}{1+b K_{f} G(s)\left(1-e^{-\tau s}\right)}
$$

The term $e^{-\tau s}$ is due to the presence of time delay in the main equation (1). For finding the critical depth of cut which puts the system in the critically stable state, the denominator of the closed loop transfer function of Eq. (4) or the characteristic equation of the system is set to zero. Namely:

$$
1+b_{\text {lim }} K_{f} G(s)\left(1-e^{-\tau s}\right)=0
$$

In which $b_{\text {lim }}$ is the critical depth of cut. In the critical state, the real part of the roots of Eq. (5) are zero and they have just imaginary part. Therefore, $s$ is set to jw. It means:

$$
1+b_{\text {lim }} K_{f} G(j w) \cdot[1-\cos (-w \tau)-j \sin (-w \tau)=0
$$

The real and imaginary parts of Eq. (6) have to be simultaneously zero. So, by considering $G(j w)=\operatorname{Re}(G(j w))+\operatorname{Im}(G(j w))$ and separating real and imaginary parts, it results:

Real part:

$$
\begin{gathered}
1+b_{\lim } K_{f}[\operatorname{Re}(G(j w))(1-\cos (w \tau))- \\
-\operatorname{Im}(G(j w)) \sin (w \tau)]=0
\end{gathered}
$$

Imaginary part:

$$
\begin{aligned}
& b_{\lim } K_{f}[\operatorname{Re}(G(j w)) \sin (w \tau)+ \\
+ & \operatorname{Im}(G(j w))(1-\cos (w \tau))]=0
\end{aligned}
$$

Then, after several mathematical manipulations the following conditions are extracted: 


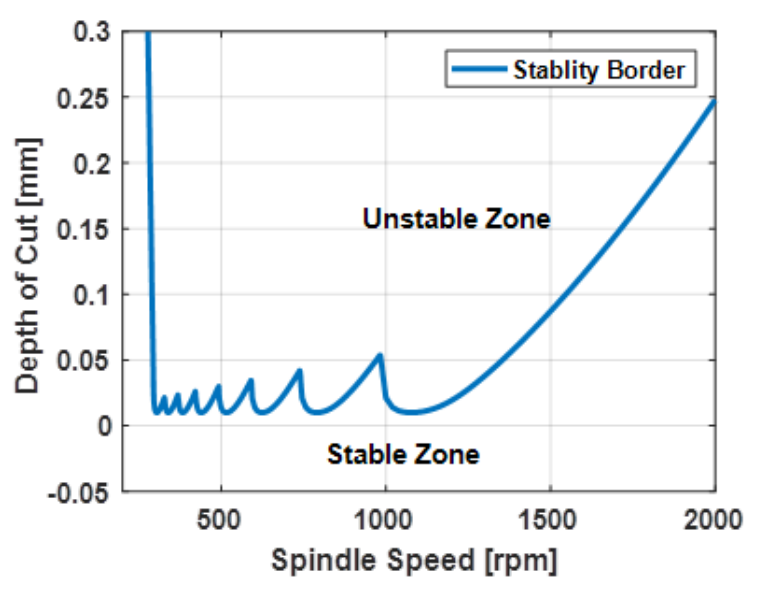

Fig. 2. A sample SLD. Lobes for different spindle speeds and depths of cut are shown. The line is the stability margin. The upper zones of the line are unstable and the lower zones are stable regions. $\mathrm{m}=140$ $\mathrm{kg}, \mathrm{c}=1689[\mathrm{~N} / \mathrm{m} . \mathrm{s}], \mathrm{k}=1.3071 \mathrm{e}+07[\mathrm{~N} / \mathrm{m}]$

$$
\begin{gathered}
b_{l i m}=\frac{1}{2 K_{f} \operatorname{Re}(G(j w))} \\
\tau=\frac{1}{\omega^{\prime}}(\varepsilon+2 k \pi) \quad N=\frac{60}{\tau} \\
\varepsilon=3 \pi+2 \times \tan ^{-1}\left(\frac{\sin (\omega \tau)}{\cos (\omega \tau)-1}\right)
\end{gathered}
$$

In Eq. (10), $\omega$ and $\varepsilon$ are the cutting tool frequency and the phase shift between the current and previous waves respectively. Also, $\mathrm{k}$ is the selected number of lobe that can be varied between 1 and the maximum spindle speed [10].

Now, according to Eqs. (9-11), the border between stable and unstable regions of the turning operation can be drawn which is called the Stability Lobe Diagram or SLD. Figure 2 shows a sample SLD. The SLD separates two regions of stable and unstable cutting operation for different spindle speed and depth of cut. If the spindle speed and depth of cut are chosen such that they locate under the lobes, the operation is stable, the finish surface is smooth, and low dynamical forces will be applied on the machine-tool. Therefore, by selecting a set of suitable spindle speed and depth of cut the chatter vibrations will be prevented.

\section{SYSTEM IDENTIFICATION}

Although, stability lobe diagram provides the possibility to predict the stability status of the turning system, this diagram is accurate only when the exact structural parameters of the system are available. However, this information is not always known or accurate because of several reasons. First of all, the impact hammer which is needed for determining structural parameters of the system might not be available especially in factory environments. Second of all, by every potential change in the process for example, changing of the tool or the workpiece, these parameters will experience alterations and they will not remain constant for every operation. And finally, even if the system parameters are provided at the beginning of the process, they might change during turning because there are many nonlinear factors included in the dynamics of the system which are not considered in the equation of motion [11].

In this paper, a method is presented based on the discrete wavelet transform (DWT) for determining the structural parameters based on the cutting force and acceleration of the tool. The main advantage of this method is that it does not need any impact hammer test. In addition, since the parameters are being measured throughout the process, they are changed continuously along with any in-process variation that might happen throughout the process.

If the dynamical equation is considered as (Eq. 1):

$$
m \ddot{y}+c \dot{y}+k y=F_{f}
$$

First DWT coefficients of $y, \dot{y}, \ddot{y}$, and $F$ are calculated, then by substituting them in Eq. (12) and solving the resulting algebraic equation, $m, c$, and $k$ can be achieved.

Therefore, for expanding discrete signals by DWT in space $l^{2}(Z)=\left\{\left.f[n]\left|\sum_{n=-\infty}^{\infty}\right| f[n]\right|^{2}<\infty \mid\right\}$, following formula can be utilized [12]:

$$
\begin{gathered}
f[n]=\frac{1}{\sqrt{M}} \sum_{k} W_{\phi}\left[j_{0}, k\right] \phi_{j_{0}, k}[n]+ \\
+\frac{1}{\sqrt{M}} \sum_{j=j_{0}}^{\infty} \sum_{k} W_{\psi}[j, k] \psi_{j, k}[n]
\end{gathered}
$$

In which $f[n]$ is the discrete signal, $\phi_{j_{n}, k}[n]$ is the scaling function, and $\psi_{j, k}[n]$ is the wavelet function. By taking the scaling and wavelet functions, the wavelet coefficients can be simply achieved by calculating the inner product of the discrete signal and scaling and wavelet functions i.e.:

$$
\begin{gathered}
W_{\phi}\left[j_{0}, k\right]=\frac{1}{\sqrt{M}} \sum_{n} f[n] \phi_{j_{0}, k}[n] \\
W_{\psi}[j, k]=\frac{1}{\sqrt{M}} \sum_{n} f[n] \psi_{j, k}[n] \quad j \geq j_{0}
\end{gathered}
$$


Eq. (4) is called approximants coefficients and Eq. (5) is called detail coefficients. The dilation version of the scaling function is determined as:

$$
\phi_{j, k}[n]=2^{\frac{j}{2}} \phi\left[2^{j} n-k\right]=\sum_{n^{\prime}} h_{\phi}\left[n^{\prime}\right] \sqrt{2} \phi\left[2\left(2^{j} n-k\right)-n^{\prime}\right]
$$

In which $h_{\phi}$ is the impulse response of a low pass filter. After some mathematical manipulations it results that the approximate coefficients can be calculated as follows which is a faster way than Eq. 14:

$$
W_{\phi}[j, k]=\left.h_{\phi}[-n] * W_{\phi}[j+1, n]\right|_{n=2 k, k \geq 0}
$$

Similarly, for detail coefficients it results:

$$
W_{\psi}[j, k]=\left.h_{\psi}[-n] * W_{\phi}[j+1, n]\right|_{n=2 k, k \geq 0}
$$

According to Eq. (12), the displacement, velocity, acceleration, and force signals are needed for determination of system parameters. Acceleration and force signals are measured by accelerometer and dynamometer sensors. But, for accessing to displacement and velocity, the acceleration signal should be integrated. It is recommended to use a noise filtering method before integration, because, probably the acceleration signal includes several noise contents and integration will lead to noise intensification. By substitution of detail and approximate coefficients of $y, \dot{y}, \ddot{y}$ and $F$ in Eq. (12), it results:

$$
\left[\begin{array}{c}
W_{\phi_{l_{1}}^{F}}^{F} \\
\vdots \\
W_{\phi_{l_{n l}}^{F}}^{F} \\
W_{\psi_{l_{1}}^{F}} \\
\vdots \\
W_{\psi_{l_{n l}}^{F}}^{F}
\end{array}\right]_{2 n_{l} \times 1}=\left[\begin{array}{ccc}
W_{\phi_{l_{1}}}^{\ddot{x}} & W_{\phi_{l_{1}}}^{\dot{x}} & W_{\phi_{l_{1}}^{x}}^{x} \\
\vdots & \vdots & \vdots \\
W_{\phi_{l_{n}}}^{\ddot{x}} & W_{\phi_{l_{n}}}^{x} & W_{\phi_{l_{n}}^{x}}^{x} \\
W_{\psi_{l_{1}}}^{\ddot{x}} & W_{\psi_{l_{1}}}^{\dot{x}} & W_{\psi_{l_{1}}^{x}}^{x} \\
\vdots & \vdots & \vdots \\
W_{\psi_{l_{n}}}^{\ddot{x}} & W_{\psi_{l_{n}}}^{\dot{x}} & W_{\psi_{l_{n}}}^{x}
\end{array}\right]_{2 n_{l} \times 3} \cdot\left[\begin{array}{c}
\widehat{M} \\
\hat{C} \\
\widehat{K}
\end{array}\right]_{3 \times 1}
$$

In Eq. (19), $W_{\phi_{1_{i}}}^{\mathrm{s}}$ and $W_{\psi_{l_{i}}}^{\mathrm{s}}$ are $\mathrm{i}$ th approximate and detail coefficients of the signal s $(y, \dot{y}$, $\ddot{y}$, or $F$ ) in the selected level of $I[13]$.

Selecting an appropriate level of decomposing is important, because those coefficients should be chosen that can properly describe the dynamics of the system in the chatter mode. Since chatter happens near the natural frequency of the lathe, and the natural freaquncy of the used apparatus is about $w_{n}$ $=400 \mathrm{~Hz}$, level two is selected. Because, the sample rate was $1000 \mathrm{~Hz}$, so the maximum frequency that can happen is $1000 \mathrm{~Hz}$. One level decomposition leads to two subspaces of 0 to $500 \mathrm{~Hz}$ and 500 to $1000 \mathrm{~Hz}$. Again, another decomposition of approximate coefficients results in two other subspaces of 0 to 250 and 250 and $500 \mathrm{~Hz}$. This level is selected because $W_{n}$ exactly lies in these frequency band.

Finally, by solving of the system of equations of Eq. (19) via least mean square method, the unknown parameters of $\hat{m}, \hat{c}$, and $\hat{k}$ can be calculated.

After determination of structural parameters, the system must be controlled in order to avoid adverse effects of chatter vibration which is described in the next section.

\section{MODEL PREDICTIVE CONTROL (MPC)}

In this work, model predictive control is used for controlling unwanted vibrations of chatter. The main goal of the Model Predictive Control is calculating the future trajectory of variable $u$ for optimization of the future behavior of the system output i.e. $y$. In this type of controller, the model of the process is used for predicting the output of the system in the future. The control effort is achieved by minimization of a cost function. Some of the merits of this controller are: 1 - using basic concepts of control in designing, 2 - simple adjusting of the controller gains, 3 - the ability to expand for complex, non-minimum phase and delayed systems easily, 4 - the ability to expand for MIMO systems, 5 - having a feedforward controller in order to compensate for measurable disturbances, 6 - easy control rule implementation, 7 - considering control signal, output, and state constraints in the design, and 8 - very useful in applications that the desired future trajectory is known.

In this research the MPC is used due to the following reasons: 
1. It can control unstable and non-minimum phase processes such as turning operation.

2. Having a forward-looking approach, this controller can avoid noises and disturbances. Since, machine-tools are placed usually in industrial environments and the sensors are exposed to different sources of noise.

3. After designing, a very simple and easy-implementing control rule will be extracted.

4. It can work in real-time applications with no trouble.

In here, SISO systems are considered, because in the turning process there is a linear second order system with one input and one output.

The state space model of system is:

$$
\begin{gathered}
x_{m}(k+1)=A_{m} x_{m}(k)+B_{m} u(k) \\
y(k)=c_{m} x_{m}(k)
\end{gathered}
$$

In which $u$ is the input, $y$ is the process output, and $\mathrm{xm}$ is the state space vector with $\mathrm{n}$ elements. In here, according to the receding horizon control principle in which just previous information is needed for predicting and control, it is assumed that the input $\mathrm{u}$ cannot directly affect the output and so $D_{m}=0$.

By considering, $\Delta x_{m}(k)=x_{m}(k)-x_{m}(k-1)$ and $\Delta u(k)=u(k)-u(k-1)$, Eq. (20) will be:

$$
\Delta x_{m}(k+1)=A_{m} \Delta x_{m}(k)+B_{m} \Delta u(k)
$$

By definition of a new set of state variables as:

$$
x(k)=\left[\Delta x_{m}(k)^{T} y(k)^{T}\right]
$$

The new state space is:

$$
\begin{gathered}
\overbrace{\left[\begin{array}{c}
\Delta x_{m}(k+1) \\
y(k+1)
\end{array}\right]}^{x(k+1)}=\overbrace{\left[\begin{array}{cc}
A_{m} & o_{m}^{T} \\
C_{m A_{m}} & 1
\end{array}\right]}^{A} \overbrace{\left.\begin{array}{c}
\Delta x_{m(k)} \\
y(k)
\end{array}\right]}^{x(k)}+\overbrace{\left[\begin{array}{c}
B_{m} \\
C_{m B_{m}}
\end{array}\right] \Delta u(k)}^{B} \\
y(k)=\overbrace{\left[\begin{array}{ll}
o_{m} & 1
\end{array}\right]}^{\Delta}\left[\begin{array}{c}
\Delta x_{m(k)}(k) \\
y(k)
\end{array}\right]
\end{gathered}
$$

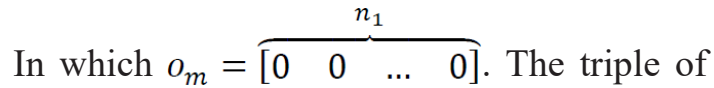
(A, B, C) in Eq. (23) is called augmented model used in model predictive control algorithm.

For computing future output based on the control future signal, $k_{i}$ is considered as the current time, and $N_{p}$ as the number of predicted future outputs called prediction horizon. This number also shows the length of the optimization window. $N_{c}$ is the control horizon which shows the number of parameters used for predicting future control trajectory. The future control inputs are: $\Delta u\left(k_{i}\right), \Delta u\left(k_{i}+1\right), \ldots, \Delta u\left(k_{i}+N_{c}-1\right)$, and future state variables are: $x\left(k_{i}+1 \mid k_{i}\right), x\left(k_{i}+2 / k_{i}\right.$ )$, \ldots, x\left(k_{i}+m / k_{i}\right), \ldots, x\left(k_{i}+N_{p} / k_{i}\right)$ in which $x\left(k i+m / k_{i}\right)$ is the predicted state variable in the time $k_{i}+m$ by possessing information of $x\left(k_{i}\right)$. Also, $N_{C} \leq N_{p}$.

By substitution of the above variables in the state space of Eq. (23), it results:

$$
\begin{aligned}
x\left(k_{i}+1 \mid k_{i}\right) & =A x\left(k_{i}\right)+B \Delta u\left(k_{i}\right) \\
x\left(k_{i}+2 \mid k_{i}\right) & =A x\left(k_{i}+1 \mid k_{i}\right)+B \Delta u\left(k_{i}+1\right) \\
& =A^{2} x\left(k_{i}\right)+A B \Delta u\left(k_{i}\right)+B \Delta u\left(k_{i}+1\right) \\
& \cdots \\
x\left(k_{i}+N_{p} / k_{j}\right) & =A^{N_{p}} X\left(k_{i}\right)+A^{N_{p}-1} B \Delta u\left(k_{i}\right)+A^{N_{p}-2} B \Delta u\left(k_{i}+1\right)+\ldots+A^{N_{p}-N_{c}} B \Delta u\left(k_{i}+N_{C}-1\right)
\end{aligned}
$$

The predicted output variables can be determined by Eq. (24) as:

$$
\begin{aligned}
& y\left(k_{i}+1 \mid k_{i}\right)=C A x\left(k_{i}\right)+C B \Delta u\left(k_{i}\right) \\
& y\left(k_{i}+2 \mid k_{i}\right)=C A^{2} x\left(k_{i}\right)+C A B \Delta u\left(k_{i}\right)+C B \Delta u\left(k_{i}+1\right) \\
& y\left(k_{i}+3 / k_{i}\right)=C A^{3} x\left(k_{i}\right)+C A^{2} B \Delta u\left(k_{i}\right)+C A B \Delta u\left(k_{i}+1\right)+C B \Delta u\left(k_{i}+2\right) \\
& y\left(k_{i}+N_{p} / k_{i}\right)=C A^{N_{p}} x\left(k_{i}\right)+C A^{N_{p}-1} B \Delta u\left(k_{i}\right)+C A^{N_{p}-2} B \Delta u\left(k_{i}+1\right)+\ldots+C A^{N_{p}-N_{c}} B \Delta u\left(k_{i}+N_{C}-1\right)
\end{aligned}
$$

If two vectors are defined as follows:

$$
\begin{aligned}
& Y=\left[y\left(k_{i}+1 \mid k_{i}\right) \quad y\left(k_{i}+2 / k_{i}\right) \quad y\left(k_{i}+3 / k_{i}\right) \quad \ldots \quad y\left(k_{i}+N_{p} / k_{j}\right)\right]_{N_{p} \times 1^{T}} \\
& \Delta U=\left[\begin{array}{llll}
\Delta u\left(k_{i}\right) & \Delta u\left(k_{i}+1\right) & \Delta u\left(k_{i}+2\right) & \Delta u\left(k_{i}+N_{c}-1\right)
\end{array}\right]_{N_{c} x 1^{T}}
\end{aligned}
$$

By compacting Eqs. (24) and (25) and considering Eq. (26), it results:

In which:

$$
Y=F_{X}\left(k_{i}\right)+\Phi \Delta U
$$




$$
F=\left[\begin{array}{c}
C A \\
C A^{2} \\
C A^{3} \\
\vdots \\
C A^{N_{p}}
\end{array}\right] ; \Phi=\left[\begin{array}{ccccc}
C B & 0 & 0 & \ldots & 0 \\
C A B & C B & 0 & \ldots & 0 \\
C A^{2} B & C A B & C B & \ldots & 0 \\
\vdots & & & & \\
C A^{N_{p}-1} B & C A^{N_{p}-2} B & C A^{N_{p}-3} B & \ldots & C A^{N_{p}-N_{C}}
\end{array}\right]
$$

The aim of horizon control is to close up the control output to the reference signal of $r\left(k_{i}\right)$ provided that the reference signal is constant in the optimization window. From this, an error function will be defined by the aim of minimizing. If the reference signal is considered as: $R_{s}^{T}=\left[\begin{array}{llll}1 & 1 & \ldots & 1\end{array}\right] r\left(k_{i}\right)$, the cost function of $\mathrm{J}$ that contains the aim of control will be:

$$
J=\left(R_{S}-Y\right)^{T}\left(R_{S}-Y\right)+\Delta U^{T} \bar{R} \Delta U
$$

The first term in Eq. (29) is related to the minimization of the error between the predicted output and the reference signal while the second term reflects the importance of $\Delta U$ magnitude in the cost function. $\bar{R}$ is a diagonal matrix in the form $\bar{R}=r_{W} I_{N_{c} \times N_{c}}\left(r_{W} \geq 0\right)$ in which $r_{W}$ should be adjusted such that the closed-loop system would have a proper performance.

The cost function of $J$ has to be minimized. For looking for the suitable $\Delta U$ that can minimize $J$, by considering Eq. (27), Eq. (29) can be rewritten as:

$$
J=\left(R_{S}-F_{X}\left(k_{i}\right)\right) T\left(R_{S}-F_{X}\left(k_{i}\right)\right)-2 \Delta U^{T} \Phi^{T}\left(R_{S}-F_{X}\left(k_{i}\right)\right)+\Delta U^{T}\left(\Phi^{T} \Phi+\bar{R}\right) \Delta U
$$

By differentiating Eq. (30) and equating to zero, the optimize answer for $\Delta U$ is achieved as:

$$
\Delta U=\left(\Phi^{T} \Phi+\bar{R}\right)^{-1} \Phi^{T}\left(R_{S}-F_{X}\left(k_{i}\right)\right)
$$

$R_{S}$ is a vector containing reference point information as $R_{S}=\overbrace{\left.\begin{array}{lllll}1 & 1 & 1 & \ldots & 1\end{array}\right]^{T}}^{N_{p}} r\left(k_{i}\right)=\bar{R}_{S} r\left(k_{i}\right)$, [14].

$\Delta U$ is the designed and optimized MPC control input which can be applied to the state space of Eq. 20.

\section{RESULTS AND DISCUSSION}

For validation of the proposed method, 25 experimental tests have been designed and conducted. In these tests, the cutting parameters are set such that the spindle speed is set to 200,600 , 1000,1400 , and $1800 \mathrm{rpm}$ and the depth of cut is set to $0.25,0.5,1,2$, and $4 \mathrm{~mm}$. All tests are performed in the wet condition. The lathe used is a CNC of type TME0. The material of workpieces is AISI1045, and each workpiece has a length of $250 \mathrm{~mm}$ and a diameter of $45 \mathrm{~mm}$. In each operation, $50 \mathrm{~mm}$ of each workpiece is machined. The soluble oil of Mobilcut100 with the concentration of $5 \%$ and the flow rate of 2 liter/min is utilized as the lubricant. The cutting tool has a ISO standard of DNMG 1506 08-PR 4225 and the tool holder model is PDJNR2020K15. For gathering tool acceleration, an accelerometer of type AC102-1A which has a sensitivity of $100 \mathrm{mV} / \mathrm{g}$ and a data logger of type 4-channel NI-USB 6009 are used. In addition, a voltage amplifier of type IEPE is employed for strengthening the output data of accelerometer. Finally, the cutting forces are collected by a dynamometer of type 9257A which is a three-axis piezoelectric Kistler dynamometer. The data gathering is performed by the sample rate of $1000 \mathrm{~Hz}$. In Fig. 3, the setup of the experiment can be seen.

\section{Real-Time System Identification}

For real-time estimation of the structural parameters of the system, the velocity and displacement signals are needed which are not directly available and are determined by integration of the tool acceleration collected by the accelerometer. In Figure 4, the acceleration and the resulting velocity and displacement signals along with the cutting force gathered by the dynamometer are shown for a sample test.

After gathering and determining $y, \dot{y}, \ddot{y}$ and $F$, their wavelet coefficients are calculated and by putting them in Eq. (12) and solving it, $m$, $c$, and $k$ are achieved. In Table 1, the estimated values of $m, c$, and $k$ are reported for all the experimental tests. Also, the stability status of each test is provided which are recognized based on the investigation of the generated chips of each test [15]. 

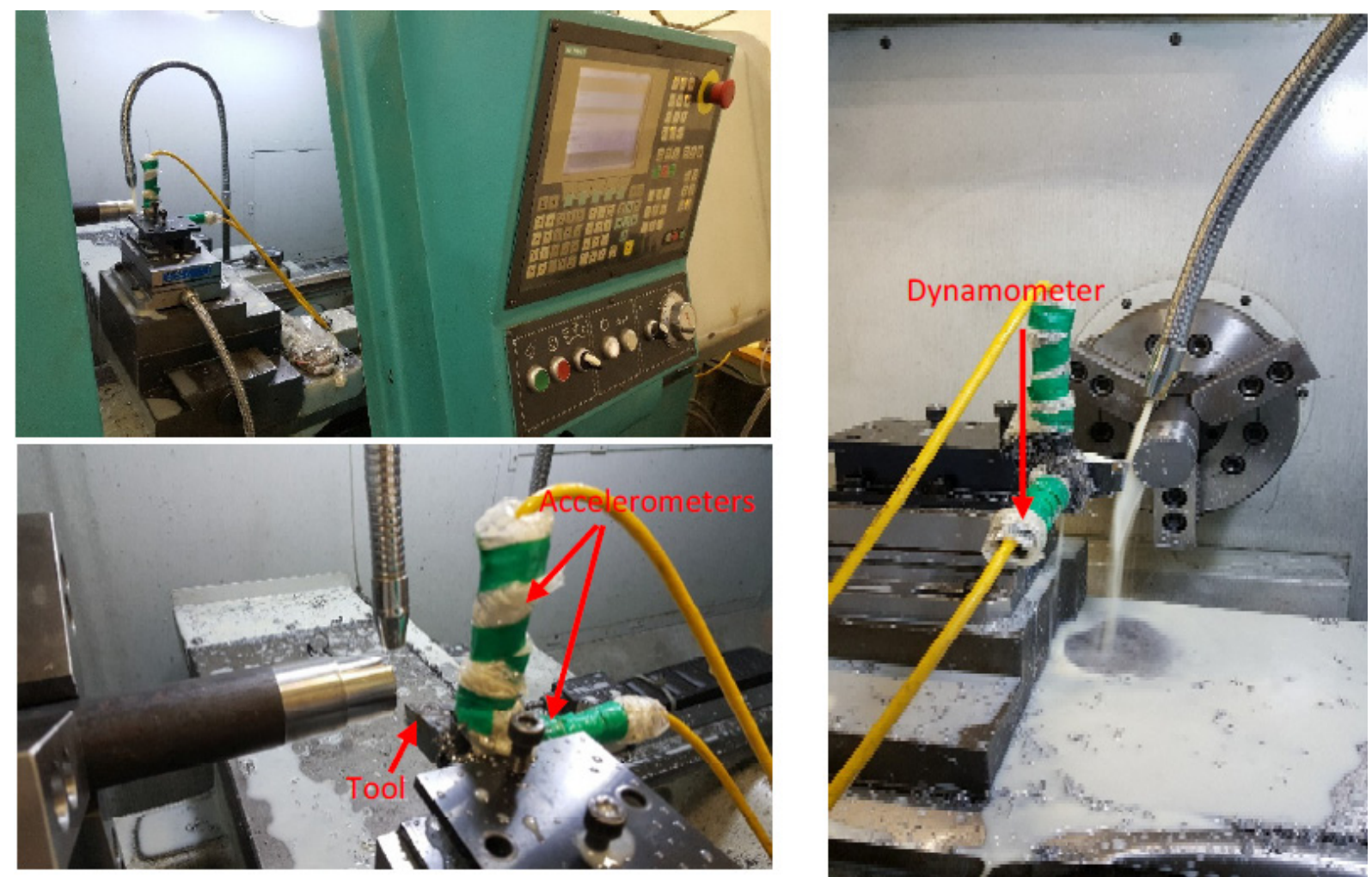

Fig. 3. The test setup showing the accelerometer, the dynamometer, the tool and the nozzle of the lubricant

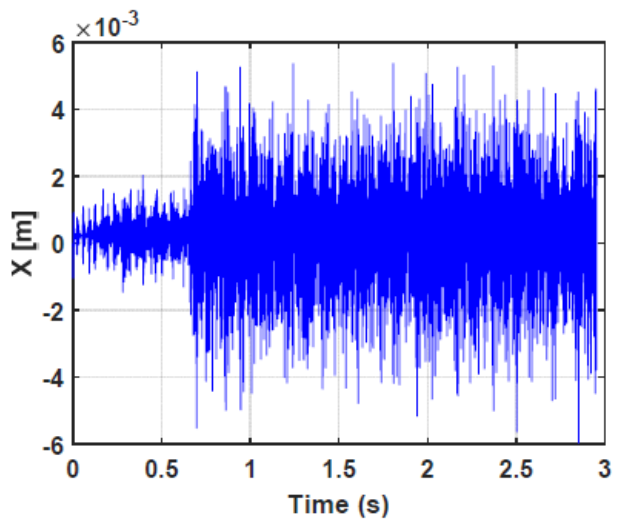

(a)

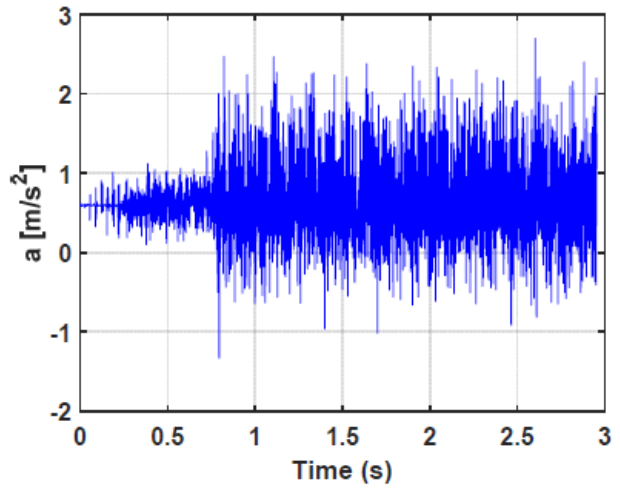

(c)

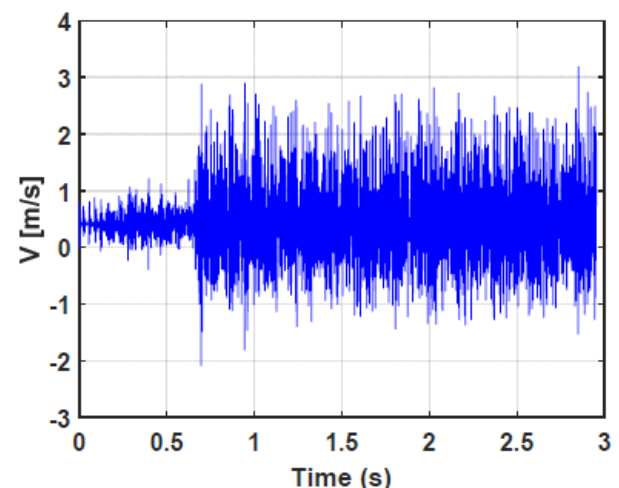

(b)

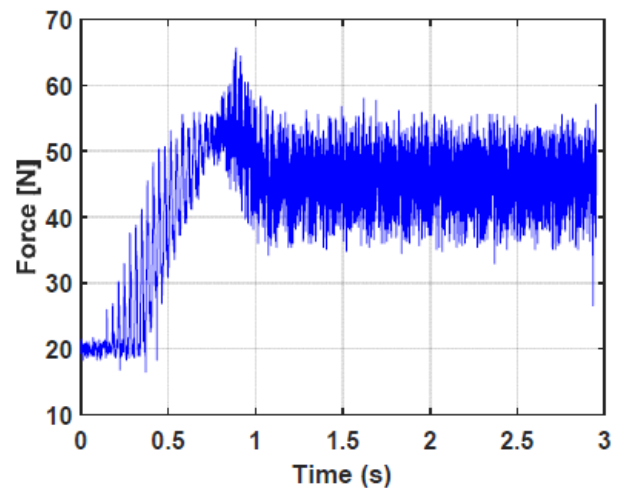

(d)

Fig. 4. The collected and estimated signals. $W=1800 \mathrm{rpm}$, depth of cut $=0.5 \mathrm{~mm}$. (a): displacement; (b): velocity; (c): acceleration; (d): force 
For the validation of the extracted structural parameters, the lob diagram of each test is plotted based on the estimated parameters, and it is investigated that whether it can correctly estimate the stability status of the operation. In Figure 5, the achieved lobe diagrams are depicted for three sample operations.

According to Table 1, the operation with the spindle speed of $200 \mathrm{rpm}$ and depth of cut of 0.5 $\mathrm{mm}$ is stable. Moreover, it can be seen that the lobe diagram shown in Fig. 5-(a) which is extracted based on the estimated structural parameters of this operation confirms it too, showing that the system identification algorithm works correctly. Similarly, for two other operations indicated in Figs. 5-(b) and (c), the algorithm has succeeded in predicting true structural parameters because the lobe diagrams truly estimate the

Table 1. The estimated values of the structural parameters

\begin{tabular}{|c|c|c|c|c|c|}
\hline w (rpm) & b $(\mathrm{mm})$ & $M(k g)$ & C (N/m.s) & $\mathrm{K}(\mathrm{N} / \mathrm{m})$ & Stability Status \\
\hline \multirow{5}{*}{200} & 0.25 & 11.2 & $3.07 e+02$ & $2.40 \mathrm{e}+06$ & Stable \\
\hline & 0.5 & 14.42 & $1.63 e+03$ & $2.96 e+06$ & Stable \\
\hline & 1 & 42.16 & $3.92 e+03$ & $8.17 e+06$ & Stable \\
\hline & 2 & 36.15 & $2.66 e+03$ & $7.08 e+06$ & Stable \\
\hline & 4 & 50.80 & $4.31 e+03$ & $1.22 \mathrm{e}+07$ & Stable \\
\hline \multirow{5}{*}{600} & 0.25 & 6.92 & $1.60 e+03$ & $2.87 e+06$ & Forced Vibration \\
\hline & 0.5 & 48.62 & $1.69 e+03$ & $7.53 e+06$ & Forced Vibration \\
\hline & 1 & 6.49 & $6.80 e+00$ & $1.27 e+06$ & Stable \\
\hline & 2 & 56.47 & $3.46 e+03$ & $1.19 e+07$ & Stable \\
\hline & 4 & 211.07 & $3.85 e+03$ & $2.08 e+07$ & Unstable \\
\hline \multirow{5}{*}{1000} & 0.25 & 15.56 & $1.09 e+03$ & $3.62 e+06$ & Stable \\
\hline & 0.5 & 35.12 & $1.44 e+03$ & $5.57 e+06$ & Marginally Stable \\
\hline & 1 & 52.09 & $1.41 e+03$ & $6.76 e+06$ & Unstable \\
\hline & 2 & 25.40 & $3.55 e+03$ & $8.73 e+06$ & Unstable \\
\hline & 4 & 41.06 & $3.05 e+03$ & $1.01 \mathrm{e}+07$ & Unstable \\
\hline \multirow{5}{*}{1400} & 0.25 & 40.25 & $4.81 e+02$ & $3.63 e+06$ & Stable \\
\hline & 0.5 & 57.67 & $3.32 e+03$ & $5.08 e+06$ & Marginally Stable \\
\hline & 1 & 34.07 & $1.61 e+03$ & $6.64 e+06$ & Unstable \\
\hline & 2 & 31.49 & $2.79 e+03$ & $8.64 e+06$ & Unstable \\
\hline & 4 & 36.88 & $4.51 \mathrm{e}+03$ & $9.21 \mathrm{e}+06$ & Unstable \\
\hline \multirow{5}{*}{1800} & 0.25 & 15.48 & $2.13 e+03$ & $3.52 e+06$ & Stable \\
\hline & 0.5 & 20.23 & $1.50 e+03$ & $5.34 e+06$ & Marginally Stable \\
\hline & 1 & 49.93 & $1.64 \mathrm{e}+03$ & $6.42 e+06$ & Unstable \\
\hline & 2 & 54.85 & $2.27 e+03$ & $8.07 e+06$ & Unstable \\
\hline & 4 & 27.85 & $6.66 e+02$ & $5.05 e+06$ & Unstable \\
\hline
\end{tabular}

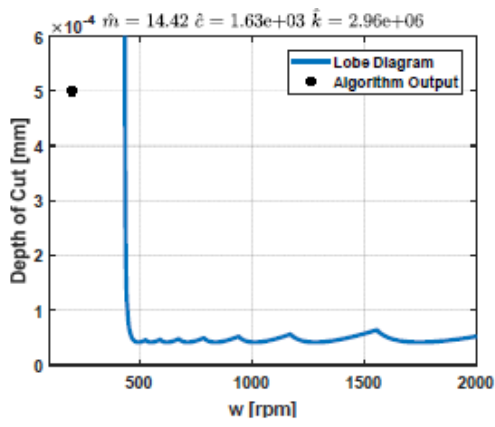

(a)

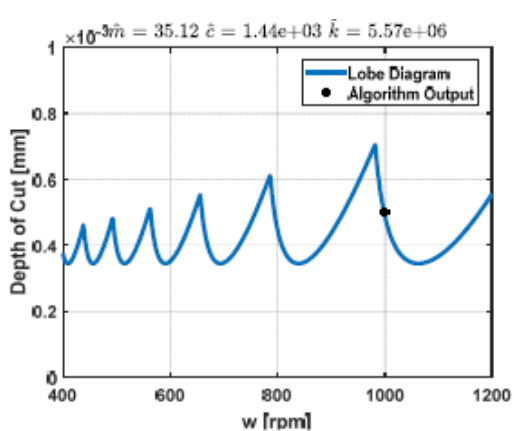

(b)

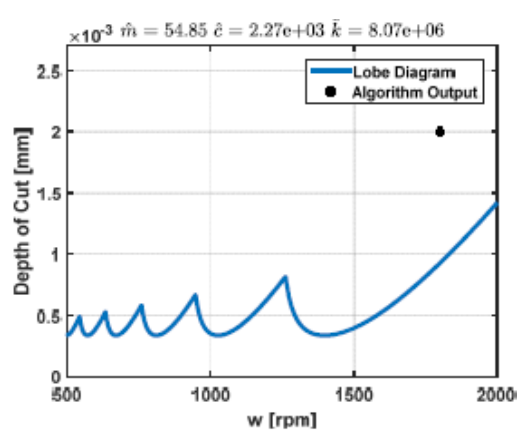

(c)

Fig. 5. The SLDs extracted based on the estimated structural parameters. (a): $w=200 \mathrm{rpm}$, depth of cut $=$ $0.5 \mathrm{~mm} ;(\mathrm{b}): \mathrm{w}=1000 \mathrm{rpm}$, depth of cut $=0.5 \mathrm{~mm} ;(\mathrm{c}): \mathrm{w}=1800 \mathrm{rpm}$, depth of cut $=2 \mathrm{~mm}$ 
status of these processes. The operations in Figs. 5-(b) and (c) are respectively marginally stable and unstable.

\section{Model Predictive Control}

After obtaining $m, c$, and $k$, the unstable operations should be controlled. The results of applying MPC on two different unstable turning processes are depicted in Figs. 6 and 7. In these figures, the displacement of the tool when there is no control algorithm and when the control algorithm is on are presented. In addition, the control effort is shown for each process. As it can be seen, the range of the displacement of the tool when there is no control algorithm is high, because the operation is in the chatter mode. On the other hand, according to Figs 6-(a) and 7-(a), after applying MPC algorithm, magnitude of the tool displacement is decreased significantly which demonstrates that MPC can effec- tively curb the unwanted vibrations of the tool. Another important point is that, the time needed for MPC algorithm to stable the process is very short and in other word, the rise and settling time is acceptable in this control algorithm. It has this advantage that before adverse influences of chatter can affect the workpiece and machine-tool, the MPC will act and force the unstable process to the stable zone.

Furthermore, the control efforts are shown in Figs. 6-(b) and 7-(b). From these figures, it is clear that the control energy needed for controlling the process is very low. This low control energy can be easily applied by the actuators such as tuned-mass-dampers, representing another important pro of MPC. Since, actuators usually have limitations on the energy that they can apply to the system. Usually, very low and very high inputs can not be applied by the actuators to the system, unless very expensive ones.

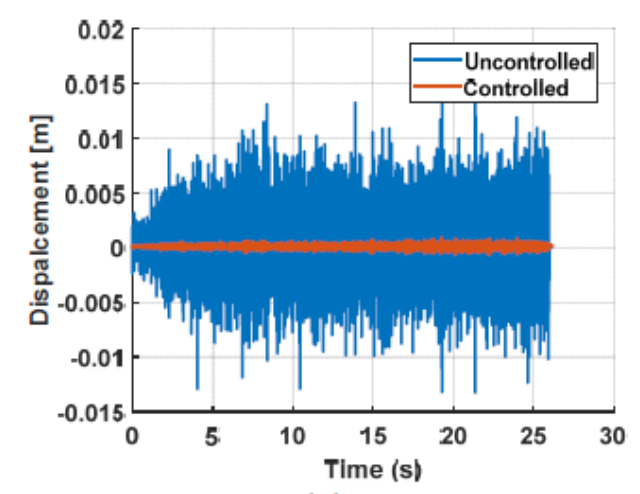

(a)

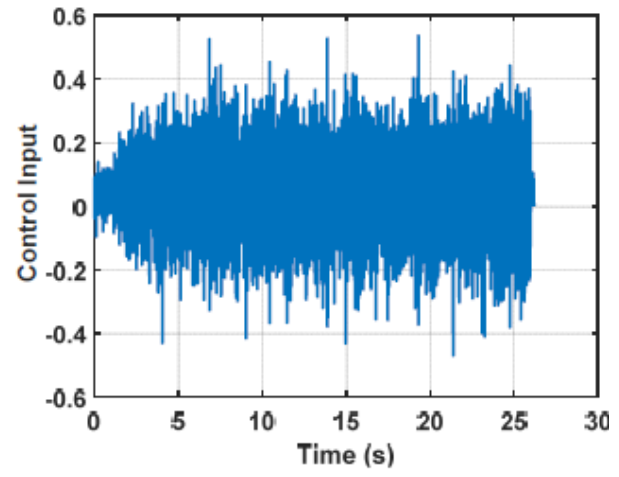

(b)

Fig. 6. The results of applying MPC. (a): The comparison between the output of the system when the control algorithm is not working and is working. (b): The control effort. $\mathrm{w}=600 \mathrm{rpm}$, depth of cut $=4 \mathrm{~mm}$

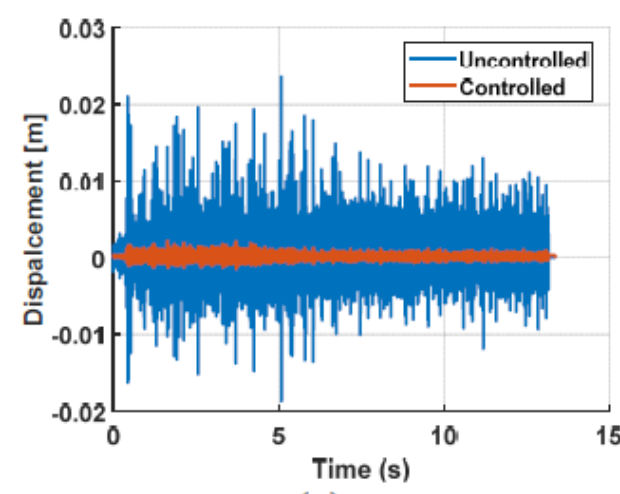

(a)

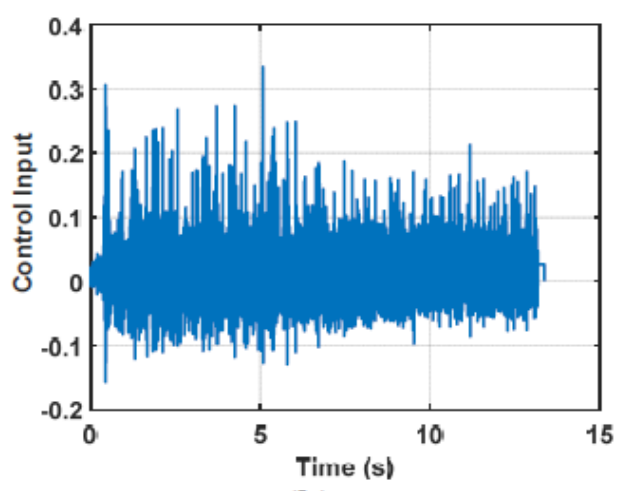

(b)

Fig. 7. The results of applying MPC. (a): The comparison between the output of the system when the control algorithm is not working and is working. (b): The control effort. $\mathrm{w}=1400 \mathrm{rpm}$, depth of cut $=1 \mathrm{~mm}$ 
For more investigation of the MPC algorithm, the performance of the MPC and PID are compared in Figs. 8 and 9. From Figs. 8-(a) and 9-(a), it is clear that the MPC can limit the unwanted vibrations of chatter more effectively than PID. For instance, in Fig. 8-(a), the maximum displacement of the tool after applying MPC is $0.44 \mathrm{~mm}$ while this parameter is $2.58 \mathrm{~mm}$ for PID. Also, maximum magnitude of the tool displacement is $0.003 \mathrm{~mm}$ for MPC and $1.61 \mathrm{~mm}$ for PID, for the process shown in Fig. 9-(a). Additionally, the control effort of MPC as it is shown in Fig. 8-(b), is between 2.12 and -2.00 , whereas for the PID is in the range of 45.94 and -39.38 . This parameter for the process depicted in Fig. 9 is between 66.50 and -64.87 for PID whereas 0.03 and -0.02 for MPC. Therefore, it can be concluded that MPC can effectively bound the unstable vibrations of tool while its control input is much lower than PID, showing the great strength and effectiveness of MPC in comparison with PID algorithm.

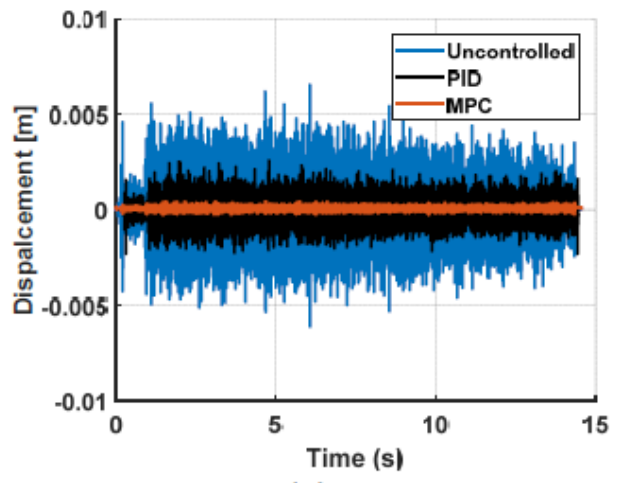

(a)

\section{CONCLUSION}

Regenerative chatter is a common phenomenon in machining process and has many destructive effects on the machine-tool and workpiece. Therefore, for preventing its adverse consequences, it is vital to control the process online. For the purpose of system control, the structural parameters of the system are needed first, while they are not usually available since some pretests such as the impact hammer test should be implemented which is not practical especially in working environments. In this work, the aim was predicting the structural parameters of the system online and control of the process based on the achieved parameters. To do this, for system identification, the wavelet coefficients of the displacement, velocity, acceleration, and force signals are calculated and then by substituting them in the system equation of motion and solving it, the structural parameters are achieved. Then, for

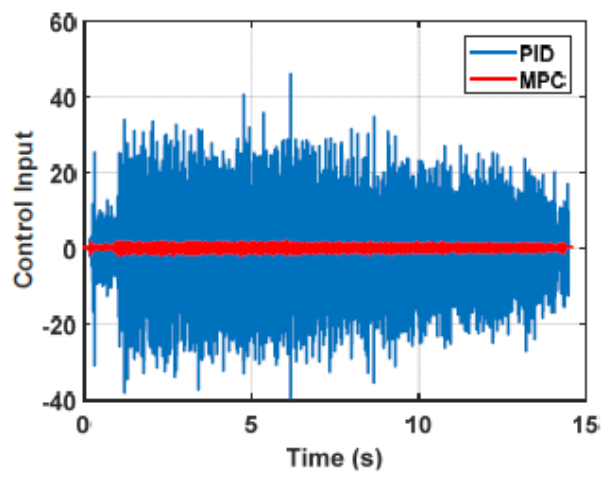

(b)

Fig. 8. The results of applying two control algorithms of PID and MPC. (a): The comparison of the tool displacement; (b): The comparison of the control effort. $\mathrm{w}=1400 \mathrm{rpm}$, depth of cut $=2 \mathrm{~mm}$

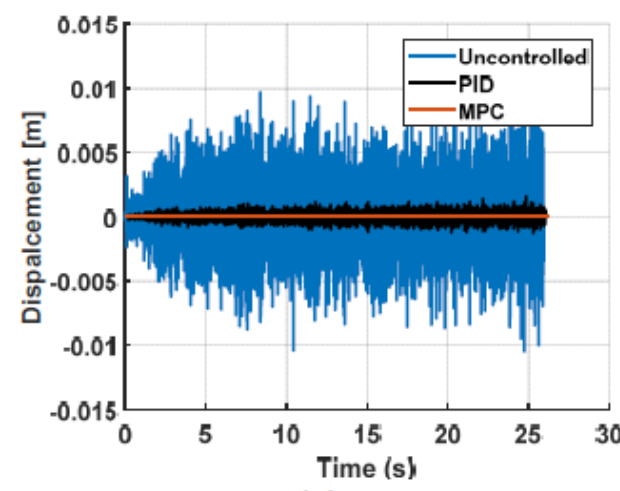

(a)

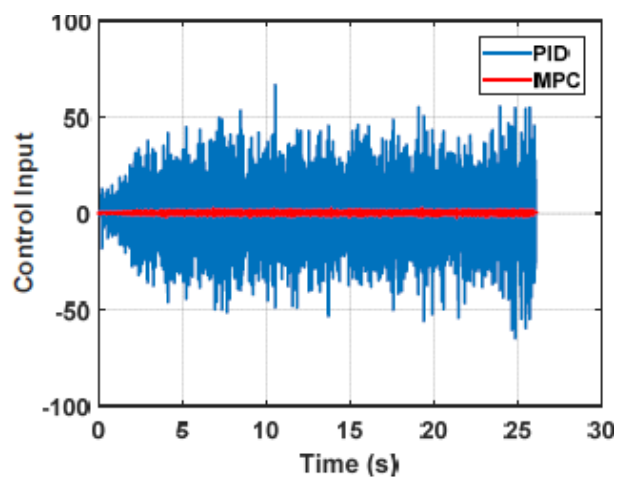

(b)

Fig. 9. The results of applying two control algorithms of PID and MPC. (a): The comparison of the tool displacement; (b): The comparison of the control effort. $\mathrm{w}=600 \mathrm{rpm}$, depth of cut $=4 \mathrm{~mm}$ 
the purpose of validation, the lobe diagrams of processes are extracted based on the estimated parameters and it is seen that they can correctly estimate the stability status of each process. After identifying systems parameters, the unstable turning processes are controlled by the MPC algorithm. For investigation of MPC performance, MPC and PID algorithms are compared. The results indicate that MPC can control the process very more accurately than PID and in the same time the control effort of MPC is much lower than PID controller which shows the superiority of the MPC over PID. Online system identification without needing any previous information, controlling the chatter phenomenon via MPC which has low delay, high performance along with low control effort are the main features of the presented method.

\section{REFERENCES}

1. Altintas, Y. and M. Weck, Chatter stability of metal cutting and grinding. CIRP annals, 2004. 53(2): 619-642.

2. Lu, K., et al., Model-based chatter stability prediction and detection for the turning of a flexible workpiece. Mechanical Systems and Signal Processing, 2018. 100: 814-826.

3. Eynian, M. and Y. Altintas, Chatter stability of general turning operations with process damping. Journal of Manufacturing Science and Engineering, 2009. 131(4): 041005.

4. Turkes, E., et al., Linear analysis of chatter vibration and stability for orthogonal cutting in turning. International Journal of Refractory Metals and Hard Materials, 2011. 29(2): 163-169.
5. Schmitz, T.-K.S., Machining Dynamics Frequency Response to Improved Productivity Springer Science+ Business Media. 2009, LLC.

6. Liu, Y., et al., Chatter reliability prediction of turning process system with uncertainties. Mechanical Systems and Signal Processing, 2016. 66: 232-247.

7. Den Hartog, J.P., Mechanical vibrations. 1985: Courier Corporation.

8. Lin, S. and M. Hu, Low vibration control system in turning. International Journal of Machine Tools and Manufacture, 1992. 32(5): 629-640.

9. Frumusanu, G.R., et al., Development of a stability intelligent control system for turning. The International Journal of Advanced Manufacturing Technology, 2013. 64(5-8): 643-657.

10. dos Santos, R.G. and R.T. Coelho, A contribution to improve the accuracy of chatter prediction in machine tools using the stability lobe diagram. Journal of Manufacturing Science and Engineering, 2014. 136(2): 021005.

11. Kurata, Y., et al., Chatter stability in turning and milling with in process identified process damping. Journal of Advanced Mechanical Design, Systems, and Manufacturing, 2010. 4(6): 1107-1118.

12. Chun-Lin, L., A tutorial of the wavelet transform. NTUEE, Taiwan, 2010.

13. Huang, S., G. Qi, and J. Yang. Wavelet for system identification. in Proceedings-Spie The International Society For Optical Engineering. 1994. Spie International Society For Optical.

14. Wang, L., Model predictive control system design and implementation using MATLAB $® .2009$ : Springer Science \& Business Media.

15. Ning, Y., M. Rahman, and Y. Wong, Investigation of chip formation in high speed end milling. Journal of materials processing technology, 2001. 113(1-3): 360-367. 